\title{
Commentary \\ Anticoagulant properties of drotrecogin alfa (activated) during hemofiltration in patients with severe sepsis
}

\author{
Anne CJM de Pont and Marcus J Schultz
}

Department of Intensive Care Medicine, Academic Medical Center, University of Amsterdam, Meibergdreef 9, 1105 AZ Amsterdam, The Netherlands

Corresponding author: Anne CJM de Pont, a.c.depont@amc.uva.nl

Published: 2 February 2009

Critical Care 2009, 13:113 (doi:10.1186/cc7684)

This article is online at http://ccforum.com/content/13/1/113

(c) 2009 BioMed Central Ltd

See related research by Camporota et al., http://ccforum.com/content/12/6/R163

\begin{abstract}
In a retrospective study among 35 severely septic patients treated with drotrecogin alfa (activated) (DrotAA) and renal replacement therapy (RRT), Camporota and colleagues demonstrated that the addition of heparin, epoprostenol, or both to DrotAA during RRT did not improve filter survival. Furthermore, in a multivariate logistic regression analysis, they identified the minimum value in platelet count as the only predictive factor of filter clotting during DrotAA infusion. These findings are in line with the previously formulated suggestion that DrotAA alone is as effective as heparin in the prevention of coagulation in the extracorporeal circuit. They also confirm the importance of baseline platelet count in the pathogenesis of extracorporeal circuit thrombosis. In the study by Camporata and colleagues, DrotAA treatment was not associated with an increase in red blood cell requirements. The results of this study supply a background to clinical decision making when choosing an anticoagulant for RRT in septic patients.
\end{abstract}

In the last 2008 issue of Critical Care, Camporota and colleagues [1] reported the results of a retrospective study analyzing filter survival time and transfusion requirements among 35 severely septic patients treated with drotrecogin alfa (activated) (DrotAA) and renal replacement therapy (RRT). DrotAA is capable of reducing mortality in severely septic patients [2] and the international guidelines for management of severe sepsis and septic shock recommend considering its use in adult patients with sepsis-induced organ dysfunction and high risk of death [3]. Among septic patients, acute renal failure (ARF) is common: its incidence ranges from $19 \%$ in moderate sepsis to $51 \%$ in septic shock [4]. ARF patients have an increased risk of mortality and this risk is even higher among patients treated with RRT [5]. As the annual incidence of sepsis ranges from 100 to 300 per 100,000 inhabitants and $30 \%$ to $40 \%$ of septic patients develop severe sepsis [6], the number of patients meeting the indications for treatment with both DrotAA and RRT is considerable.
As DrotAA is an anticoagulant itself, the risk of bleeding during its use might be increased by the addition of other anticoagulants during RRT. Until now, only one report of three cases has been published on this topic. This report suggested that Drot AA alone is as effective as heparin in the prevention of coagulation in the extracorporeal circuit [7].

The current study demonstrates that the addition of heparin, epoprostenol, or both to DrotAA during RRT does not prolong filter survival. The lack of an additional effect of heparin on filter survival is not surprising since the antithrombotic effect of DrotAA is not enhanced by the addition of heparin [8]. However, since epoprostenol is a potent inhibitor of platelet function and DrotAA does not seem to have a direct inhibitory effect on platelet aggregation [9], one might have expected prolongation of filter survival during treatment with both DrotAA and epoprostenol.

Notably, multivariate logistic regression analysis identified the minimum value in platelet count as the only predictive factor of filter clotting during DrotAA infusion. Several studies have demonstrated the association between baseline platelet count and circuit clotting, especially during postdilution $[10,11]$. It is hypothesized that, due to an increase in local viscosity and shear stress, a higher baseline platelet count facilitates platelet cohesion along the hollow fiber wall [11]. Increased platelet cohesion may cause enhanced thrombin generation in the hemofilter, leading to filter clotting. During sepsis, however, coagulation is initiated by inflammatory mediators, such as endotoxin and cytokines, capable of inducing tissue factor expression on monocytes and macrophages [12]. DrotAA can completely inhibit tissue factorinduced platelet activation [13]. Since epoprostenol inhibits platelet function by increasing the synthesis of cyclic adenosine monophosphate, its effect may be insufficient to

$A R F=$ acute renal failure; $\operatorname{Drot} A A=$ drotrecogin alfa (activated) RRT $=$ renal replacement therapy. 
add to the effect of coagulation-induced platelet consumption in combination with complete inhibition of tissue factorinduced platelet activation by DrotAA.

In the current study, no difference in red blood cell requirements, either between DrotAA episodes and post-DrotAA episodes or between medical and surgical patients, was found. This is surprising given that, among the 4,459 patients included in INDEPTH (International Integrated Database for the Evaluation of Severe Sepsis and Drotrecogin alfa [activated] Therapy), the bleeding incidence in surgical patients was about 10 times higher in the DrotAA group than in the placebo group $(4.9 \%$ versus $0.5 \%)$ and in medical patients it was about 2.5 times higher (2.6\% versus $1 \%$ [ [14]. The lack of a difference in the current study might be due to the relatively small number of patients.

\section{Conclusion}

In summary, the results of the current study add to the understanding of the effect of Drot AA on coagulation in the extracorporeal circuit and supply a background to clinical decision making when choosing an anticoagulant for RRT in septic patients.

\section{Competing interests}

The authors declare that they have no competing interests.

\section{References}

1. Camporota L, Corno E, Menaldo E, Smith H, Lei K, Beale R, Wyncoll D: Filter survival and blood products requirement in patients with severe sepsis receiving drotrecogin alfa (activated) and requiring renal replacement therapy. Crit Care 2008, 12:R163.

2. Bernard GR, Vincent JL, Laterre PF, LaRosa SP, Dhainaut JF, Lopes-Rodriguez A, Steingrub JS, Garber GE, Helterbrand JD, Ely EW, Fisher CJ Jr. for the Recombinant Human Activated Protein C Worldwide Evaluation in Severe Sepsis (PROWESS) study group: Efficacy and safety of recombinant human activated protein C for severe sepsis. N Engl J Med 2001, 344:699-709.

3. Dellinger RP, Levy MM, Carlet JM, Bion J, Parker MM, Jaeschke R, Reinhart K, Angus DC, Brun-Buisson C, Beale R, Calandra T, Dhainaut JF, Gerlach H, Harvey M, Marini JJ, Marshall J, Ranieri M, Ramsay G, Sevransky J, Thompson T, Townsend S, Vender JS, Zimmerman JL, Vincent JL for the International Surviving Sepsis Campaign Guidelines Committee: Surviving Sepsis Campaign: international guidelines for management of severe sepsis and septic shock: 2008. Crit Care Med 2008, 36:296-327.

4. Schrier RW, Wang W: Acute renal failure and sepsis. $N$ Eng/ J Med 2004, 351:159-169.

5. Payen D, de Pont AC, Sakr Y, Spies C, Reinhart K, Vincent JL for the Sepsis Occurrence in Acutely III Patients (SOAP) Investigators: A positive fluid balance is associated with a worse outcome in patients with acute renal failure. Crit Care 2008, 12:R74.

6. Esteban A, Frutos-Vivar F, Ferguson ND, Peñuelas O, Lorente JA, Gordo F, Honrubia T, Algora A, Bustos A, García G, DiazRegañón IR, Ruiz de Luna R: Sepsis incidence and outcome: contrasting the intensive care unit with the hospital ward. Crit Care Med 2007, 35:1284-1289.

7. de Pont AC, Bouman CS, de Jonge E, Vroom MB, Büller HR, Levi $M$ : Treatment with recombinant human activated protein $C$ obviates additional anticoagulation during continuous venovenous hemofiltration in patients with severe sepsis. Intensive Care Med 2003, 29:1205.

8. Levi M, Levy M, Williams MD, Douglas I, Artigas A, Antonelli M, Wyncoll D, Janes J, Booth FV, Wang D, Sundin DP, Macias WL for the Xigris and Prophylactic HepaRin Evaluation in Severe Sepsis (XPRESS) Study Group: Prophylactic heparin in patients with severe sepsis treated with drotrecogin alfa (activated). Am J Respir Crit Care Med 2007, 176:483-490.

9. Schuerholz T, Friedrich L, Marx G, Kornau I, Sümpelmann R, Scheinichen D: Effect of drotrecogin alfa (activated) on platelet receptor expression in vitro. Platelets 2007, 18:373-378.

10. de Pont AC, Oudemans-van Straaten HM, Roozendaal KJ, Zandstra DF: Nadroparin versus dalteparin anticoagulation in highvolume, continuous venovenous hemofiltration: a double-blind, randomized crossover study. Crit Care Med 2000, 28:421-425.

11. de Pont AC, Bouman CS, Bakhtiari K, Schaap MC, Nieuwland R, Sturk A, Hutten BA, de Jonge E, Vroom MB, Meijers JC, Büller HR: Predilution versus postdilution during continuous venovenous hemofiltration: a comparison of circuit thrombogenesis. ASA/O J 2006, 52:416-422.

12. Esmon CT: Crosstalk between inflammation and thrombosis. Maturitas 2004, 47:305-314.

13. Kaiser B, Jeske W, Hoppensteadt DH, Walenga JM, Drohan W, Fareed J: In vitro studies on the effect of activated protein $\mathrm{C}$ on platelet activation and thrombin generation. Thromb Res 1997, 87:197-204.

14. Payen D, Sablotzki A, Barie PS, Ramsay G, Lowry S, Williams MD, Sarwat S, Northrup J, Toland P, Booth FV: International integrated database for the evaluation of severe sepsis and drotrecogin alfa (activated) therapy: analysis of efficacy and safety data in a large surgical cohort. Surgery 2007, 141:548561. 